\title{
The Improvement on the Maximum Mutual Information Image Registration
}

\author{
Zhao Xiaolei \\ Weinan Teachers University \\ Weinan Shaanxi, China \\ LUOZXL@163.com
}

\begin{abstract}
Keywords: Maximum mutual information; Wavelet decomposition; Image registration
\end{abstract}
\begin{abstract}
This paper studies the theory and algorithm based on the maximum mutual information image registration, And on that basis, the paper comes up with an idea of algorithm improvement to combine the wavelet decomposition with the theory and algorithm. This paper also proves from the theory and practice that the improved algorithm is superior to maximum mutual information image registration. In this paper, we can learn that the effect of the maximum mutual information image registration based on two layers of wavelet transformation is the best of all by comparing the different experimental dates.
\end{abstract}

\section{Introduction}

Nonrigid registration is an important technique in medical image processing[1]. However, in general, it requires a large computation time, which is a big disadvantage for many clinical applications. Mutual information is a basic conception of the information theory[2], which represents the pertinency measurement of two random variable statistics. Collignon、Wells and other experts use mutual information as the measurement of multimoding medical image registration.

When the registration of two images is processed by using mutual information as the measurement of the similarity of the two images, their corresponding image character mutual information should be the maximal if the two images shared the same structure reach the best registration. This paper presents the image registration method based on the combination of wavelet changes and mutual information and validates its efficiency and advantages through experiments.

\section{Nonrigid Registration Method}

This section describes the various components of the nonrigid registration method. The design of the algorithm is largely based on the papers by Rueckert et al., Mattes et al., and Thévenaz and Unser. The registration method uses cubic B-splines to parameterize the deformation field, and mutual information as the similarity measure.[3]

In the statistics the gray scale of the corresponding pixels of the identical object is not absolute but corelative, which is the foundation of image registration based on mutual information.

The registration of the two images by using mutual information as the measurement of the similarity based on the following principle:

$\alpha^{*}=\arg \max _{T} \alpha(R(x, y), s(F(x, y))$

When the two images share the identical target or the target structure reaches the optimal registration, the mutual information of their corresponding image feature will be the maximum.

\section{Optimization methods}

The PV(Partial Volume) interpolation[4] is used to get the statistics coalesce histogram and caculate the mutual information of the reference image $\mathrm{R}$ and the relocating image $\mathrm{F}$, then Powell algorithem, the direction accelerated method, is used to judge whether the parameter is optimal according to the 
maximum mutual information theory. If it is not optimal, the search for superior parameter still goes on. when searching, the process - space geometric transformation (affine) - statistics coalesce histogram(PV interpolation) - to calculate the mutual information value - to judge the optimization - is repeated continuously till the parameters which meet the precision standard are found, and finally the registration parameter is outputted.

\section{The method of image registration based on wavelet decomposition and maximum mutual information}

\section{The principle of image registration based on wavelet decomposition}

The principle that the sampling is obtained every two values is used in the wavelet filter[5], so we suppose that the obtaine image is $\{f(2 m, 2 n)\}$ after the original image is sampled every two values backwards. The original image position is $\left\{f\left(2 m^{\prime}, 2 n^{\prime}\right)\right\}$, the low-pass filtered coefficient images are $\left\{c^{M}(m, n)\right\}$ and $\left\{c^{M}\left(m^{\prime}, n^{\prime}\right)\right\}, \mathbf{T}$ is affine Transformation, $T m=m^{\prime}$ and $T n=n^{\prime}$. The coordinate transformation of rotation transformation and translation transformation before and after the wavelet filter is studied in the following part.

Rotation transformation: if $\mathrm{T}$ is supposed to be the rotation transformation in the original image, it transfers the image $\left\{c^{M+1}(2 m, 2 n)\right\}$ into $\left\{c^{M+1}(2 m \cos \theta+2 n \sin \theta,-2 m \sin \theta+2 n \cos \theta)\right\}$, but after wavelet low-pass filter, $c^{M+1}(2 m \cos \theta+2 n \sin \theta,-2 m \sin \theta+2 n \cos \theta)$ changes into:

$$
\begin{aligned}
& \sum_{k, l}\{h[(k-2 m) \cos \theta+(l-2 n) \sin \theta] \times h[-(k-2 m) \sin \theta+(l-2 n) \cos \theta] \\
& \left.\times c^{M+1}(k \cos \theta+l \sin \theta,-k \sin \theta+l \cos \theta)\right\} \\
& =\sum_{k, l}\{h[(k \cos \theta+l \sin \theta)-2(m \cos \theta+n \sin \theta)] \times h[(-k \sin \theta+l \cos \theta) \\
& \left.-2(-m \sin \theta+n \cos \theta)] \times c^{M+1}(k \cos \theta+l \sin \theta,-k \sin \theta+l \cos \theta)\right\} \\
& =\sum_{p, q} h[p-2(m \cos \theta+n \sin \theta)] \times h[q-2(-m \sin \theta+n \cos \theta)] \times c^{M+1}(p, q) \\
& =c^{M}(m \cos \theta+n \sin \theta,-m \sin \theta+n \cos \theta)
\end{aligned}
$$

and $p=k \cos \theta+l \sin \theta, q=-k \sin \theta+l \cos \theta \quad$ The above function shows that the rotation transformation of the two images can be transferred into the rotation registration of the approximate weight of the two images after the wavelet decomposition. At the same time the rotation angle of the two original images is equal to that of the two approximate weigh images after their decomposition.

translation transformation: If $\mathrm{T}$ is the translation transformation, it is supposed to transform the original image $\{f(2 m, 2 n)\}$ or $\left\{c^{M+1}(2 m, 2 n)\right\}$ into $\left\{c^{M+1}(2 m+2 \Delta x, 2 n+2 \Delta y)\right\}$. In order to get the relations between the image that obtained by wavelet transform and low-pass filter and its approximate weight, the transformation is as follows: suppose $p=k-2 m, q=l-2 n$ then we get

$$
\begin{aligned}
& \sum_{k, l} h(k-2 m) h(l-2 n) c^{M+1}(k, l) \\
& =\sum_{p, q} h(p) h(q) c^{M+1}(2 m+p, 2 n+q)
\end{aligned}
$$

therefore after the wavelet transform and low-pass filter $\left\{c^{M+1}(2 m+2 \Delta x, 2 n+2 \Delta y)\right\}$ is transferred

$$
\sum_{p, q} h(p) h(q) c^{M+1}(2 m+2 \Delta x+p, 2 n+2 \Delta y+q)
$$

into $=\sum_{p, q} h(p) h(q) c^{M+1}(2(m+\Delta x)+p, 2(n+\Delta y)+q)$

$$
=C^{M}(m+\Delta x, n+\Delta y)
$$

The above function shows that the translation transformation of the two images can be transferred into the translation registration of the approximate weight of the two images after the wavelet decomposition. If the translation of the two images transformation is $(2 \Delta x, 2 \Delta y)$, the 
translation of the approximate weight of the two images after their separate decomposition is $(\Delta x, \Delta y)$.

The above reasoning proves that the rotation registration and the tranlation registration of two images can be transferred into the rotation registration and the tranlation registration of the approximate weight [6] of the two images after the wavelet decomposition. This way can save the calculation amount when evaluating the registration parameter. If the size of the original image is $N \times N$ and the calculation amount[7] when evaluating the registration parameter is $\mathrm{K}$, in theory the size of the low frequency image after wavelet decomposition is $N / 2 \times N / 2$ and the calculation amount is $K / 4$. This can efficiently save the calculation amount and promote the registration speed.

The realization of registration algorithm based on wavelet decomposition and maximum mutual information

$\mathrm{R}$ is supposed to be the reference image and $\mathrm{F}$ is the image that will be registered, so the registration process is as follows:

The planar wavelet decomposition is used to decompose the image $R(x, y)$ and $F(x, y)$ seperately for several times. $L L_{R 0}(x, y)$ is the image $\mathrm{R}$ and $L L_{F 0}(x, y)$ is the image $\mathrm{F} . \quad L L_{R j}(x, y)$ is the j-layer approximate weight of $\mathrm{R}$ and $L L_{F j}(x, y)$ is the j-layer approximate weight[8] of $\mathrm{F}$, in this process $\mathrm{j}=0,1, \ldots \mathrm{N}$.

The approximate weights after the highest layer wavelet decomposition of image $\mathrm{R}$ and $\mathrm{F}$ are put into the original points, then PV interpolation is used to calculate the mutual information of $L L_{R N}(x, y)$ and $L L_{F N}(x, y)$.

Powell algorithm, the direction acceleration method[9], is used to evaluate whether the parameter is superior based on the maximum mutual information. If it is not superior, in each dimension Brent algorithm is used to iteratively search and estimate the registration parameter to increase the mutual information continuously[10].

The registered parameter is $(\theta, \Delta x, \Delta y)$.

The registration output is $\left(\theta, 2^{N} \times \Delta x, 2^{N} \times \Delta y\right)$.

\section{The result and analysis of the experiment}

To validate the correctness of the algorithm, the right direction of the coordinate axis $\mathrm{X}$ is set to be positive, the downward direction of the coordinate axis $\mathrm{Y}$ is set to be positive, the clockwise of the rotation direction is positive. The experiment is shown in figure two, the registered image is tupian2.bmp(image A in figure 1), image B is obtained after tupian2.bmp translate ten units pixel and float ten units angle along the positive direction of axis $\mathrm{X}$ and $\mathrm{Y}$ through the maximum mutual information registration algorithm. $\mathrm{t}=164.4019 \mathrm{~s}$ ( $\mathrm{t}$ is the time that is used for registration). $\mathrm{x}=9.5027(\mathrm{x}$ is the value of translation on axis $\mathrm{X}$ ), $\mathrm{y}=11.2884$ ( $\mathrm{y}$ is the value of translation on axis $\mathrm{Y}$ ), the rotation $\theta=11.3891$. In other words, if the unit pixel of the translation of image $\mathrm{B}$ on axis $\mathrm{X}$ is $\mathrm{x}=10.5027$, the unit pixel of the translation of image $\mathrm{B}$ on axis $\mathrm{Y}$ is $\mathrm{y}=10.2884$ and the angle of the contrarotation is $\theta=11.3891$, image $\mathrm{B}$ and image A can reach approximate registration. The time needed for the registration is $\mathrm{t}=154.4019 \mathrm{~s}$.

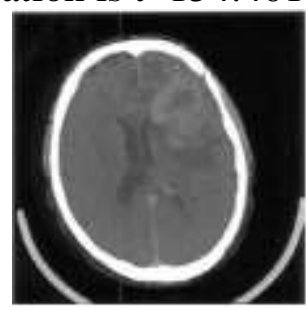

Figure A

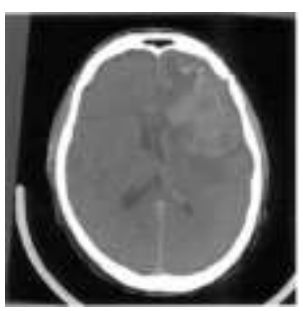

Figure B

Figure 1 the Experimental Results

The process of image registration based on wavelet decomposition and maximum mutual 
information is the same as previous. Wavelet decomposition is applied before the image is registered. The input parameter is the same as the previous, the result is as follows after different layers of wavelet decomposition is used (the time includes the time used for wavelet decomposition).

the result of the first layor wavelet decomposition is $t=50.4901 \mathrm{~s}, x=10.6898, y=11.1195$, $\theta=10.9005$

the result of the second layor wavelet decomposition is $t=21.2563 \mathrm{~s}, x=10.0854, y=10.9618$, $\theta=10.0996$

the result of the third layor wavelet decomposition is $t=6.7995 \mathrm{~s}, x=9.3269, y=11.1667, \theta=9.0753$

Error computing: (actual value- return value) /actual value $\times 100 \%$, the actual value is a certain value given before registration, the return value is the absoulte value of the return value after registration, all errors are shown in Table 1:

Table 1 method error( pencentage)

\begin{tabular}{cccc}
\hline & $\begin{array}{c}\mathrm{X} \\
\text { direction } \\
\text { error }\end{array}$ & $\begin{array}{c}\text { Y } \\
\text { direction } \\
\text { error }\end{array}$ & $\begin{array}{c}\text { Rotation } \\
\text { error }\end{array}$ \\
$\begin{array}{c}\text { Basic } \\
\text { registration } \\
\text { first layor }\end{array}$ & 4.973 & 2.884 & 3.891 \\
$\begin{array}{c}\text { decomposition } \\
\text { second layor } \\
\text { decomposition } \\
\text { third layor t } \\
\text { decomposition }\end{array}$ & 3.102 & 1.195 & 0.995 \\
\hline
\end{tabular}

The analysis curve of method error is shown as figure 2:

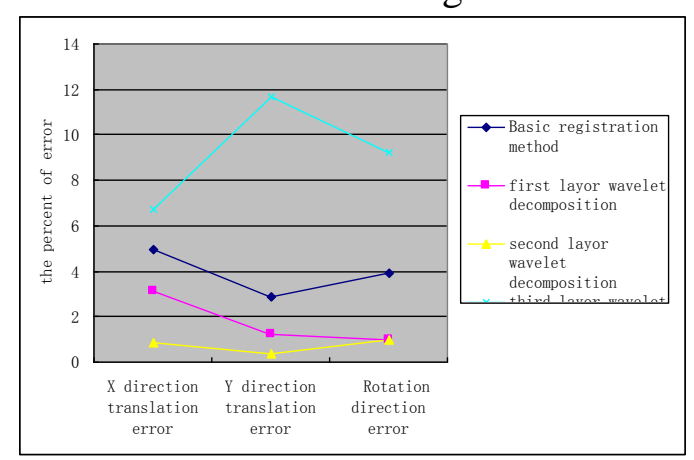

Figure 2. The analysis curve of method error

Through the previous results, we can see the registration introduced in the paper is far superior than the registration based on the maximum mutual information in time. According to figure 2 , the combination of wavelet decomposition and maximum mutual information can control the error in certain limit and reach better registration result.

\section{Conclusion}

The theory of applying the maximum mutual information into the image registration shows the disadvantages of longer registration time and low accuracy. Based on this theory the algorithm of combining wavelet decomposition and maximum mutual information is proposed and deduced in theoretically in this paper. As the experimental datas show that the image registration algorithm based on wavelet decomposition and maximum mutual information proposed in this paper can improve the registration speed effectively and shows superior registration effect. 


\section{Acknowledgements}

Project in mathematics funded by Shaanxi Province(project number:14XZD011)

Project funded by Education Department of Shaanxi Provincial Government (project umber:16JK1272)

\section{References}

[1] Xiaogang Du, Jianwu Dang, Yang ping Wang, et al.2013. Mutual information medical image registration based on firefly algorithm, Computer Science, 40(7):273-276

[2] Wang Min, Song Tengyi, Remote Sensing Image Retrieval by Scene Semantic Matching[J],IEEE Transactions on Geoscience and Remote Sensing, 2013, 51(5):2874-2886.

[3] MaJinfu.Multi-source Image Fusion Technology and Application Research[D].Xi'an: Xi'an University Science and Technology,2009.

[4] Zhang Defeng. The Process of Digital Image based on MATLAB[M].BeiJing: Machine Industry Press, 2009.

[5] Xia, R., Zhao, J. and Liu, Y., 2013, October. A robust feature-based registration method of multimodal image using phase congruency and coherent point drift. In Eighth International Symposium on Multispectral Image Processing and Pattern Recognition, International Society for Optics and Photonics, (2013).

[6] Dame A., Marchand E. 2012. Second-order optimization of mutual information for real-time image registration, IEEE Transactions on Image Processing, 21(9):4190-4203.

[7] Li Wang, Min Wang. 2015. Infrared image registration algorithm based on multi-parameter segmentation mutating method. Application Research of Computers, 33.

[8] Xia Zhu, Renwen Chen, Piaoyan Zhang. 2015.Application of cultural improved particle swarm optimization in image registration. Application Research of Computers, 32(6): 1874-1877.

[9] Hou M, Chen C, Tang D, Luo S, Yang F, Gu N. Magnetic microbubble-mediated ultrasound-MRI registration based on robust optical flow model. Biomed Eng Online. 2015;14(Suppl 1):S14.

[10] Ye, Yuanxin, Lian Xiong and Jie Shan.: Automated Multi-Source Remote Sensing Image Registration Based on Phase Congruency. ISPRS-International Archives of the Photogrammetry, Remote Sensing and Spatial Information Sciences, 1, pp. 189-194, (2012). 